\title{
Power Generation Limits in Thermal, Chemical and Electrochemical Systems
}

\author{
S. Sieniutycz \\ Faculty of Chemical and Process Engineering, Warsaw University of Technology, \\ 1 Waryńskiego Street, 00-645 Warsaw (Poland) \\ Phone 00-48-22-256340; fax: 00-48-22-251440, e-mail sieniutycz@ichip.pw.edu.pl
}

\begin{abstract}
Power generation limits are evaluated via optimization for various energy converters, such like thermal, solar, chemical, and electrochemical engines, in particular fuel cells. Thermodynamic analyses lead to converters' efficiencies, which help to solve problems of optimal upgrading and downgrading of resources. While methods of static optimization, i.e. differential calculus and Lagrange multipliers, are sufficient for steady processes, dynamic optimization applies the variational calculus and dynamic programming for unsteady processes. In reacting systems chemical affinities constitute prevailing components of an overall efficiency, thus flux balances are applied to derive power in terms of active parts of chemical affinities. Methodological similarity is observed when treating power limits in flow thermal machines and fuel cells. The examples show power maxima in fuel cells and prove suitability of a thermal machine theory to chemical and electrochemical systems. The main novelty of contribution in the fuel cell context consists in introducing an effective change of Gibbs free energy between products $p$ and reactants $s$ which takes into account lowering of voltage and power caused by the incomplete conversion of the overall electrochemical reaction.
\end{abstract}

\section{Key words}

Power limits, entropy, exergy, chemical engines, fuel cells.

\section{Introduction}

In a previous work [1] we discussed models of power production and its limits in purely thermal systems with finite rates. In particular, radiation engines were analyzed as important nonlinear systems governed by laws of thermodynamics and transport phenomena. Temperatures $T$ of participating media were only necessary variables to describe these systems. In the present work we treat generalized systems in which both temperatures $T$ and chemical potentials $\mu^{k}$ are essential. This is associated with engines propelled by fluxes of both energy and substance. In a process of power production shown in Fig. 1 two media differing in values of $T$ and $\mu$ interact through an energy generator (engine), and the process is propelled by diffusive and/or convective fluxes of heat and mass transferred through 'conductances' or boundary layers. The energy flux (power) is created in the generator between the resource fluid ('upper' fluid 1) and, say, an environment fluid ('lower' fluid, 2).

Both transfer mechanisms and values of conductances of boundary layers influence the rate of power production [2]-[5]. Local fluxes of heat and power do not change along the process path only when both reservoirs (streams) in Fig.1 are infinite.
When one, say, upper, reservoir is finite, its thermal potential decreases along the stream path, which is the consequence of the energy balance. Any finite reservoir is thus a resource reservoir. It is the resource property or the finiteness of amount or flow of a valuable substance or energy which changes the upper fluid properties along its path. Then, in the engine mode, one observes fluid's relaxation to the equilibrium with an infinite lower reservoir, usually the environment. This is a cumulative effect obtained for a resource fluid at flow, a set of sequentially arranged engines, and an infinite bath [6]. An inverse process, which needs the supply of an external power, may be referred to the upgrading of the resource in a heat pump [7]. From the optimization viewpoint, these cases refer to dynamical processes, or sequence of states, either in the chronological time or in holdup (spatial) time. Studies of resource downgrading or upgrading apply methods of dynamical optimization [8].

Downgrading or upgrading of resources may occur also in electrochemical systems of fuel cell type. Fuel cells working in power production mode are electrochemical flow engines propelled by chemical reactions. Their performance is determined by magnitudes and directions of participating streams and by mechanism of electric current generation. Voltage lowering in fuel cells below the reversible value is a good measure of their imperfection which influences the downgrading and upgrading of reagents. Yet, in this paper we restrict to the steady-state fuel cell systems.

Section 2 of the present paper derives suitable controls in power systems, the so called Carnot variables. These results are common for all processes considered here. Thermal systems are treated in Sects 3-8 of this paper, whereas chemical and electrochemical systems (fuel cells) are analyzed in Sects 9 and 10. Section 11 presents basic conclusions.

\section{Carnot Controls in Power Yield Systems.}

Diverse controls can be applied in power systems to represent the propelling fluxes of heat and mass transfer and accomplish the task of a sustainable energy conversion. Here we shall recall and use definitions of Carnot control variables whose derivations and applications were originated in our previous work, $[9,10]$. We begin with the simplest case of no mass transfer, i.e. we shall consider a steady, internally reversible ('endoreversible') heat engine with a perfect internal power 
generator characterized by temperatures of circulating fluid $T_{1}$, and $T_{2}$, Fig.1. Stream temperatures, attributed to the bulk o each fluid are $T_{1}$ and $T_{2}$. The inequalities $T_{1}>T_{1},>T_{2},>T_{2}$ are valid for engine mode of the system. The internal entropy balance yields

$$
\frac{q_{2}}{T_{2}}=\frac{q_{1}}{T_{1^{\prime}}}
$$

Continuity of pure heat fluxes through each boundary layer (each conductor) is assumed $\left(q_{1}=q_{1}\right.$, and $q_{2}=q_{2}$ ), the property which does not hold in the case when heat transfer is coupled with transfer of substances. As a flux can be normalized versus a constant mass flux we neglect dots over symbols of fluxes.

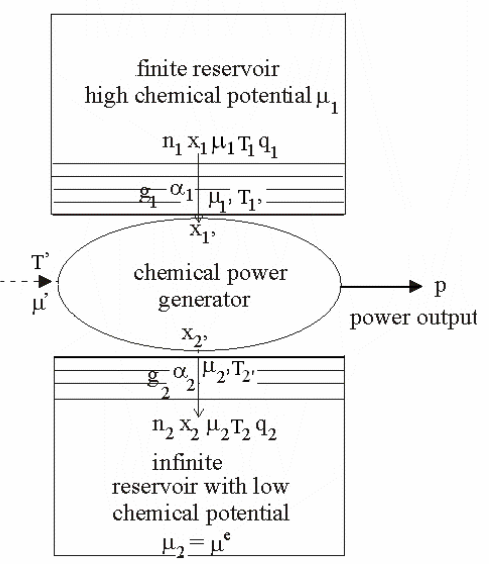

Fig.1. A scheme of chemical and/or thermal engine.

Total entropy balance of the system leads to total entropy source $\sigma_{\mathrm{s}}$ as the difference of outlet and inlet entropy fluxes

$$
\sigma_{s}=\frac{q_{2}}{T_{2}}-\frac{q_{1}}{T_{1}}=\frac{q_{1}}{T_{2}} \frac{T_{2^{\prime}}}{T_{1^{\prime}}}-\frac{q_{1}}{T_{1}}=\frac{q_{1}}{T_{2}}\left(\frac{T_{2^{\prime}}}{T_{1^{\prime}}}-\frac{T_{2}}{T_{1}}\right) .
$$

With an effective temperature called Carnot temperature

$$
T^{\prime} \equiv T_{2} \frac{T_{1^{\prime}}}{T_{2^{\prime}}}
$$

entropy production of the endoreversible process, Eq. (2), is

$$
\sigma_{s}=q_{1}\left(\frac{1}{T^{\prime}}-\frac{1}{T_{1}}\right)
$$

This form is identical with the familiar expression obtained for processes of purely dissipative heat exchange between two bodies with temperatures $T_{1}$ and $T^{\prime}$.

From the entropy and energy balances of an internally reversible process the endoreversible thermal efficiency follows in terms of temperatures of the circulating fluid

$$
\frac{p}{q_{1}}=\eta=1-\frac{T_{2^{\prime}}}{T_{1^{\prime}}}
$$

In terms of temperature $T$ ' of Eq. (3) this efficiency assumes the classical Carnot form containing the temperature in the bulk of the second reservoir and temperature $T$ '.

$$
\eta=1-\frac{T_{2}}{T^{\prime}}
$$

This property substantiates the name "Carnot temperature" for control variable $T$ '. When a control action takes place, the superiority of Eq. (6) over Eq. (5) consists in using in (6) single, free control $T$, instead of two constrained controls of Eq. (5) (linked by an internal balance of the entropy). Moreover, the endoreversible power is also of classical form

$$
p=\eta q_{1}=\left(1-\frac{T_{2}}{T^{\prime}}\right) q_{1}
$$

Importantly, the derivation of Eqs. (1) - (7) does not require any specific assumptions on the nature of heat transfer. In terms of $T$ ' description of thermal endoreversible cycles is broken down to formally "classical" equations which contain $T$ ' in place of $T_{1}$. In irreversible situations Carnot temperature $T$ ' efficiently represents temperature of the upper reservoir, $T_{1}$. Yet, at the reversible Carnot point, where $T_{1},=T_{1}$ and $T_{2},=T_{2}$, Eq. (3) yields $T^{\prime}=T_{1}$, thus returning to the classical reversible theory. These properties of Carnot temperature render descriptions of endoreversible and reversible cycles similar. They also make the variable $T^{\prime}$ a suitable control in static and dynamic cases $[9,10]$.

For the purpose of this paper it is worth knowing that in terms of $T$ ' the linear heat transfer is described by a simple equation

$$
q_{1}=g\left(T_{1}-T^{\prime}\right) \text {, }
$$

where $g$ is overall heat transfer conductance i.e. the product of a total exchange area and an overall heat transfer coefficient [8]. For a linear resource relaxing to the thermodynamic equilibrium along the stationary Lagrangian path or for an unsteady relaxation, the kinetics related to Eq. (8) has the linear form

$$
\frac{d T_{1}}{d \tau}=T^{\prime}-T_{1}
$$

where the non-dimensional time $\tau$ satisfies Eq. (38) below and is related to the overall conductance $g$ of Eq. (8). Subscript 1 is neglected in equations describing dynamical paths. The resource (or an "upper stream") is upgraded whenever Carnot temperature $T$ ' is higher than resource's temperature $T_{1}$. It is downgraded (relaxes to the thermodynamic equilibrium with an infinite "lower" stream or the environment of $T=T_{2}$ ) when Carnot temperature $T$ ' is lower than resource's temperature $T_{1}$. In linear systems, power-maximizing $T^{\prime}$ is proportional to the resource's temperature $T_{1}$ at each time instant [6]). For two finite streams with constant heat capacities, see [11].

The notion of Carnot temperature can be extended to chemical systems where also the Carnot chemical potential emerges. The structure of Eq. (1) also holds to systems with mass transfer provided that instead of pure heat flux $q$ the so called total heat flux (mass transfer involving heat flux) $Q$ is introduced

$$
Q \equiv q+T s_{1} n_{1}+\ldots T s_{k} n_{k} . .+T s_{m} n_{m}
$$

or, since the heat flux equals the difference between total energy flux $\varepsilon$ and flux of enthalpies of transferred components, $q=\varepsilon-h$,

$$
Q \equiv \varepsilon-\mu_{1} n_{1} \ldots \mu_{k} n_{k} \ldots-\mu_{m} n_{m} \equiv \varepsilon-G
$$

where $G$ is the flux of Gibbs function. The equality

$$
\varepsilon=Q+G
$$

is fundamental in the theory of chemical engines; it indicates that power can be generated by two propelling fluxes: heat flux $Q$ and Gibbs flux $G$, each generation having its own efficiency (thermal and chemical efficiencies). The related driving forces are the temperature difference and chemical affinity.

When mass transfer is included the internal entropy balance of the perfect engine has in terms of total heat flux $Q$ the same structure as Eq. (1) 


$$
Q_{2} / T_{2^{\prime}}=Q_{1} / T_{1^{\prime}}
$$

The continuity of energy and mass fluxes through the resistive layers leads to 'primed' fluxes in terms of those for the bulk. Assuming a complete conversion we restrict to power yield by a simple reaction $A_{1}+A_{2}=0$ (isomerisation or phase change of $A_{1}$ into $\mathrm{A}_{2}$ ). The energy balance

$$
\varepsilon_{1}=\varepsilon_{2}+p
$$

and the mass balance in terms of conserved fluxes through cross-sections 1' and 1 as well as 2' and 2

$$
n_{1}=n_{2}
$$

are combined with Eq. (13) describing the continuity of the entropy flux in the reversible part of the system. This yields

$$
\frac{\varepsilon_{1}-\mu_{1^{\prime}} n_{1}}{T_{1^{\prime}}}=\frac{\varepsilon_{2}-\mu_{2^{\prime} n_{2}}}{T_{2^{\prime}}}
$$

Eliminating $\varepsilon_{2}$ and $n_{2}$ from these equations yields

$$
\frac{\varepsilon_{1}-\mu_{1^{\prime} n_{1}}}{T_{1^{\prime}}}=\frac{\varepsilon_{1}-p-\mu_{2^{\prime} n_{1}}}{T_{2^{\prime}}}
$$

whence

$$
\frac{p}{T_{2^{\prime}}}=\frac{\varepsilon_{1}-\mu_{2^{\prime} n_{1}}}{T_{2^{\prime}}}-\frac{\varepsilon_{1}-\mu_{1^{\prime}} n_{1}}{T_{1^{\prime}}} .
$$

which leads to a power expression

$$
p=\varepsilon_{1}-\varepsilon_{2}=\varepsilon_{1}\left(1-\frac{T_{2^{\prime}}}{T_{1^{\prime}}}\right)+T_{2^{\prime}}\left(\frac{\mu_{1^{\prime}}}{T_{1^{\prime}}}-\frac{\mu_{2^{\prime}}}{T_{2^{\prime}}}\right) n_{1}
$$

In Eq. (19) power $p$ is expressed in terms of fluxes continuous through the conductors.

Entropy production in the system follows form the balance of fluxes in the bulks of the streams

$$
\sigma_{s}=\frac{q_{2}}{T_{2}}-\frac{q_{1}}{T_{1}}+\left(s_{2}-s_{1}\right) n_{1}
$$

Eliminating $q_{2}$ from this result with the help of the energy balance (14) we obtain

$$
\sigma_{s}=\left(q_{1}+h_{1} n_{1}\right)\left(\frac{1}{T_{2}}-\frac{1}{T_{1}}\right)+\left(\frac{\mu_{1}}{T_{1}}-\frac{\mu_{2}}{T_{2}}\right) n_{1}-\frac{p}{T_{2}} .
$$

An equivalent form of this equation is the formula

$$
p=\varepsilon_{1}\left(1-\frac{T_{2}}{T_{1}}\right)+T_{2}\left(\frac{\mu_{1}}{T_{1}}-\frac{\mu_{2}}{T_{2}}\right) n_{1}-T_{2} \sigma_{s}
$$

which may be compared with the same power evaluated for the endoreversible part of the system

$$
p=\varepsilon_{1}\left(1-\frac{T_{2^{\prime}}}{T_{1^{\prime}}}\right)+T_{2^{\prime}}\left(\frac{\mu_{1^{\prime}}}{T_{1^{\prime}}}-\frac{\mu_{2^{\prime}}}{T_{2^{\prime}}}\right) n_{1} .
$$

The comparison of Eqs (22) and (23) yields an equality

$$
\begin{aligned}
& \varepsilon_{1}\left(1-\frac{T_{2}}{T_{1}}\right)+T_{2}\left(\frac{\mu_{1}}{T_{1}}-\frac{\mu_{2}}{T_{2}}\right) n_{1}-T_{2} \sigma_{s} \\
& =\varepsilon_{1}\left(1-\frac{T_{2^{\prime}}}{T_{1^{\prime}}}\right)+T_{2^{\prime}}\left(\frac{\mu_{1^{\prime}}}{T_{1^{\prime}}}-\frac{\mu_{2^{\prime}}}{T_{2^{\prime}}}\right) n_{1}
\end{aligned}
$$

from which the entropy production can be expressed in terms of bulk driving forces and active driving forces. We obtain

$$
\sigma_{s}=\frac{\varepsilon_{1}}{T_{2}}\left(\frac{T_{2^{\prime}}}{T_{1^{\prime}}}-\frac{T_{2}}{T_{1}}\right)+n_{1}\left(\frac{\mu_{1}}{T_{1}}-\frac{T_{2^{\prime}}}{T_{2}}\left(\frac{\mu_{1^{\prime}}}{T_{1^{\prime}}}-\frac{\mu_{2^{\prime}}}{T_{2^{\prime}}}\right)-\frac{\mu_{2}}{T_{2}}\right)
$$

This expression generalizes Eq. (3) for the case when a single reaction $A_{1}+A_{2}=0$ undergoes in the system. Equation (25) leads again to the definition of Carnot temperature in agreement with Eq. (3) and to Carnot chemical potential of the first component

$$
\frac{\mu^{\prime}}{T^{\prime}}=\frac{\mu_{2}}{T_{2}}+\frac{T_{2^{\prime}}}{T_{2}}\left(\frac{\mu_{1^{\prime}}}{T_{1^{\prime}}}-\frac{\mu_{2^{\prime}}}{T_{2^{\prime}}}\right) .
$$

In a special case of an isothermal process the above formula yields a chemical control variable

$$
\mu^{\prime}=\mu_{2}+\mu_{1^{\prime}}-\mu_{2^{\prime}}
$$

which has been used earlier to study an isothermal engine [12]. After introducing the Carnot temperature in accordance with Eq. (3), total entropy production of the endoreversible power generation by the simple reaction $A_{1}+A_{2}=0$ (isomerisation or phase change of $A_{1}$ into $A_{2}$ ), takes the following simple form

$$
\sigma_{s}=\varepsilon_{1}\left(\frac{1}{T^{\prime}}-\frac{1}{T_{1}}\right)+\left(\frac{\mu_{1}}{T_{1}}-\frac{\mu^{\prime}}{T^{\prime}}\right) n_{1} .
$$

Introducing the above formula total heat $Q_{1}$ satisfying $Q_{1} \equiv \varepsilon_{1}-\mu_{1} n_{1}$ we finally obtain

$$
\sigma_{s}=Q_{1}\left(\frac{1}{T^{\prime}}-\frac{1}{T_{1}}\right)+n_{1} \frac{\mu_{1}-\mu^{\prime}}{T^{\prime}},
$$

where $Q_{1}=q_{1}+T_{1} s_{1} n_{1}$ is the total heat flux propelling the power generation in the system. The resulting equation is formally equivalent with a formula obtained for the purely dissipative exchange of energy and matter between two bodies with temperatures $T_{1}$ and $T^{\prime}$ and chemical potentials $\mu_{1}$ and $\mu$ '.

\section{Steady State Energy Systems}

Carnot variables $T^{\prime}$ and $\mu^{\prime}$ are two free, independent control variables applied in power maximization of steady and dynamical generators. Ideas referring to endoreversible systems may be generalized to those with internal dissipation.
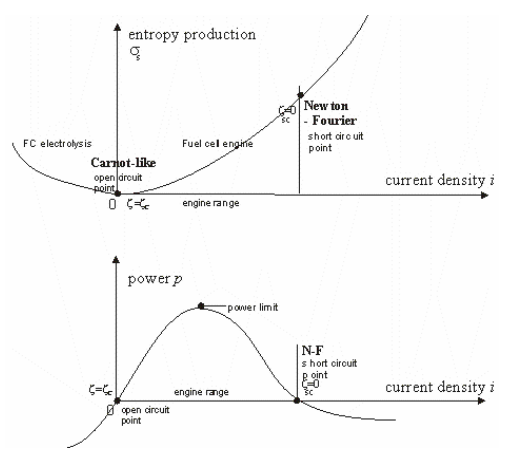

Fig. 2. Qualitative picture illustrating entropy production and power yield in fuel cells in terms of the density of electric current. For thermal engines the picture is qualitatively similar when the electric current is replaced by the entropy flux.

Majority of research papers on power limits published to date deals with systems in which there are two infinite reservoirs. To this case refer steady analyses of Chambadal-Novikov-CurzonAhlborn engine (CNCA engine; [2]) in which energy exchange is described by the Newton cooling law, or of the StefanBoltzmann engine, a system with the radiation fluids and energy flow governed by the Stefan-Boltzmann law [3]. Entropy 
production and power diagrams for these systems are shown in Fig. 3. In a CNCA engine the maximum power point may be related to the optimum value of a free control variable which may be efficiency $\eta$, heat flux $q_{1}$, or Carnot temperature $T$ '. When internal irreversibilities within the generator play a role, a pseudo-Carnot formula applies instead of Eq. (6), in which $T_{2}$ is replaced by product $\Phi T_{2}$, where $\Phi$ is the internal irreversibility factor [5]. In terms of bulk temperatures $T_{1}, T_{2}$ and $\Phi$ one finds at the maximum power point

$$
T_{o p t}^{\prime}=\left(T_{1} \Phi T_{2}\right)^{1 / 2} .
$$

For the Stefan-Boltzmann engine exact expression at the optimal point cannot be determined analytically, yet, the temperature can be found graphically from the chart $p=f\left(T^{\prime}\right)$. A pseudo-Newtonian model [5], [7], which treats state dependent energy exchange with coefficient $\alpha\left(T^{3}\right)$, omits to a considerable extent analytical difficulties of the Stefan-Boltzmann equation.

\section{Dynamical Energy Generation}

Dynamical energy yield requires the knowledge of an extremal curve rather than an extremum point. This leads us to variational metods (to handle extrema of functionals) in place of static optimization methods (to handle extrema of functions). Observed non-exponential shape of the relaxation curve is the consequence of nonlinear properties of the radiation fluid. Nonexponential are also other curves describing the radiation relaxation, e.g. those following from exact models involving the Stefan-Boltzmann equation [4], [5], [7]. Optimal, powermaximizing $T(t)$ is accompanied by optimal control $T^{\prime}(t)$; they both are components of the dynamic optimization solution.

Energy limits of dynamical processes are inherently connected with exergies, the classical exergy and its rate-dependent extensions. To obtain the classical exergy from work functionals it suffices to assume that the thermal efficiency of the system is identical with the Carnot efficiency. On the other hand, nonCarnot efficiencies, influenced by rates, lead to 'generalized exergies'. The benefit from generalized exergies is that they define stronger energy limits than classical ones [8].

\section{Finite Resources and Finite Rate Exergies}

Two different kinds of work, first associated with the resource downgrading during its relaxation to the equilibrium and the second - with the reverse process of resource upgrading, are essential. This idea is illustrated in Fig. 2 of ref [1]. In engine mode work is released, in heat-pump mode work is supplied. Optimal work follows as a general potential depending on end states and duration. For appropriate boundary conditions the function of extremum work coincides with the exergy at flow as the function that characterizes quality of resources.

Total power obtained from an infinite number of infinitesimal stages representing the resource relaxation is determined as the Lagrange functional of the following structure

$$
\dot{W}\left[\mathbf{T}^{i}, \mathbf{T}^{f}\right]=\int_{t^{i}}^{t^{f}} f_{0}\left(T, T^{\prime}\right) d t=-\int_{t^{i}}^{t^{f}} \dot{G} c(T) \eta\left(T, T^{\prime}\right) \dot{T} d t
$$

where $f_{0}$ is power generation intensity, $\dot{G}$ - resource flux, $c(T)$ specific heat, $\eta\left(T, T^{\prime}\right)$ - efficiency in terms of state $T$ and control $T$, further $\mathbf{T}$ - enlarged state vector comprising state and time, $t$ - time variable (residence time or holdup time) for a resource contacting with energy transfer surface. For a constant mass flux of a resource stream, one can extremize power per unit mass flux, i.e. the quantity of specific work dimension called 'work at flow'. A non-dimensional time $\tau$ is often used in the description

$$
\tau \equiv \frac{x}{H_{T U}}=\frac{\alpha^{\prime} a_{v} F}{\dot{G} c} x=\frac{\alpha^{\prime} a_{v} F v}{\dot{G} c} t=\frac{t}{\chi}
$$

This definition assures that $\tau$ is identical with the number of the energy transfer units, and related to system's constants, $\chi$ and $H_{\mathrm{TU}}$ (relaxation constant and height of the transfer unit). Equation (31), which links both times, $\tau$ and $t$, contains resource's flow $\dot{G}$, stream velocity $v$ through cross-section $A^{\perp}$, and heat transfer exchange surface per unit volume $a_{\mathrm{v}}$ [5]. Function $f_{0}$ in Eq. (30) contains thermal efficiency, $\eta$, described by a practical counterpart of the Carnot formula. When $T>T^{e}$, efficiency $\eta$ decreases in the engine mode below $\eta_{\mathrm{C}}$ and increases in the heat-pump mode above $\eta_{\mathrm{C}}$. At the limit of vanishing rates $d T / d t=0$ and $T^{\prime} \rightarrow T$. Work of each mode simplifies then to the classical exergy.

Solutions to work extremum problems can be obtained by variational methods, i.e. via Euler-Lagrange equation of variational calculus. However, such solutions do not contain direct information about the optimal work function $V=$ $\max (\dot{W} / \dot{G})$. Yet, this function can be obtained by solving the related Hamilton-Jacobi-Bellman equation (HJB equation: [8] and [13]). For the Newtonian energy transfer (linear kinetics)

$$
\frac{\partial V}{\partial \tau}-\max _{T^{\prime}}\left\{\left(-\frac{\partial V}{\partial T}-c\left(1-\frac{T^{e}}{T^{\prime}}\right)\right)\left(T^{\prime}-T\right)\right\}=0
$$

Extremum work function $V=\max (\dot{W} / \dot{G}$ in equations of this type is a function of the final state and total duration.

After evaluation of optimal control and its substitution to Eq. (32) one obtains a nonlinear equation

$$
\left.\frac{\partial V}{\partial \tau}-c\left\{\sqrt{T^{e}}-\sqrt{T\left(1+c^{-1} \partial V / \partial T\right.}\right)\right\}^{2}=0
$$

which is the Hamilton-Jacobi equation of the problem. Its solution can be found by the integration of work intensity along an optimal path, between limits $T$ and $T^{\mathrm{f}}$. A reversible (path independent) part of $V$ is the classical exergy $A\left(T, T^{\mathrm{e}}, 0\right)$.

When analytical difficulties are serious method of dynamic programming is applied to solve a discrete HJB equation which is in, fact, Bellman's equation of dynamic programming for cascades [13]. Modeling of multistage power yield in sequences of engines is discussed in the previous publications [5] and [11].

\section{Selected HJB Equations for Energy Systems}

We shall display some Hamilton-Jacobi-Bellman equations, in particular for radiation power systems. A suitable example is a radiation engine whose power integral is approximated by a 
pseudo-Newtonian model of radiative energy exchange. The model is associated with an optimal function

$$
V\left(T^{i}, t^{i}, T^{f}, t^{f}\right) \equiv \max _{T^{\prime}(t)}\left(-\int_{t^{i}}^{t^{f}} \dot{G}_{m} c_{m}\left(1-\Phi^{\prime} \frac{T^{e}}{T^{\prime}}\right) v\left(T^{\prime}, T\right) d t\right)
$$

where $v=\alpha\left(T^{3}\right)\left(T^{\prime}-T\right)$. Alternative forms use expressions of Carnot temperature $T$ ' in terms of other control variables [5]. Optimal power (34) can be referred to a pseudolinear kinetics $\mathrm{d} T / \mathrm{d} t=f\left(T, T^{\prime}\right)$ consistent with rate $v=\alpha\left(T^{3}\right)\left(T^{\prime}-T\right)$. A general form of HJB equation for work function $V$ is

$$
-\frac{\partial V}{\partial t}+\max _{T^{\prime}(t)}\left(f_{0}\left(T, T^{\prime}\right)-\frac{\partial V}{\partial T} f\left(T, T^{\prime}\right)\right)=0,
$$

where $f_{0}$ is defined as the integrand in Eq. (34).

A more exact model or radiation conversion relaxes the assumption of the pseudo-Newtonian transfer and applies the Stefan-Boltzmann law. For the symmetric model of radiation conversion (both reservoirs composed of radiation we obtain

$$
\dot{W}=\int_{t^{i}}^{t^{f}} \dot{G} c(T)\left(1-\frac{\Phi T^{e}}{T^{\prime}}\right) \beta \frac{T^{a}-T^{\prime a}}{\left(\Phi^{\prime}\left(T^{\prime} / T^{e}\right)^{a-1}+1\right) T^{a-1}} d t .
$$

Here $\Phi^{\prime} \equiv \Phi \mathrm{g}_{1} / \mathrm{g}_{2}$ and coefficient $\beta=\sigma a_{v} c_{h}^{-1}\left(p_{m}^{0}\right)^{-1}$ is related to molar constant of photons density $p_{m}^{0}$ and Stefan-Boltzmann constant $\sigma$. Power exponent $a=4$ for radiation and $a=1$ for a linear resource. With a dynamical state equation [5]

$$
\frac{d T}{d t}=-\beta \frac{T^{a}-T^{\prime a}}{\left(\Phi^{\prime}\left(T^{\prime} / T^{e}\right)^{a-1}+1\right) T^{a-1}}
$$

applied in general Eq. (35) we obtain a HJB equation

$$
\frac{\partial V}{\partial t}=\max _{T^{\prime}(t)}\left\{\left(\dot{G}_{c}\left(1-\Phi \frac{T^{e}}{T^{\prime}}\right)+\partial V / \partial T\right) \beta \frac{T^{a}-T^{\prime a}}{\left(\Phi^{\prime}\left(T^{\prime} / T_{2}\right)^{a-1}+1\right) T^{a-1}}\right\}
$$

Dynamics (37) is the characteristic equation to Eq. (38).

For a hybrid model of the radiation conversion (upper reservoir composed of the radiation and lower reservoir of a Newtonian fluid, power is

$$
\dot{W}=-\int_{\tau^{i}}^{t^{f}} G_{c}(T)\left(1-\frac{\Phi T^{e}}{T^{\prime}}\right) u d t
$$

whereas the related Hamilton-Jacobi-Bellman equation is

$$
-\frac{\partial V}{\partial t^{f}}+\max _{T^{\prime}(t)}\left\{-\left(\dot{G}_{c}(T)\left(1-\frac{\Phi T^{e}}{T^{\prime}}\right)+\frac{\partial V}{\partial T^{f}}\right) u\right\}=0
$$

where by definition:

$$
T^{\prime} \equiv\left(T^{a}+\beta^{-1} T^{a-1} u\right)^{1 / a}+\Phi \beta^{-1} T^{a-1} u g_{1} / g_{2}
$$

is Carnot temperature of this particular problem [5].

\section{Some Solutions of HJB Equations}

Expressions extremized in HJB equations are some Hamiltonians. Pontryagin's variable for the energy problem is $z$ $=-\partial V / \partial T$. Applying feedback control optimal temperature $T$ ' or some other control is implemented as the quantity maximizing the hamiltonian with respect to Carnot temperature at each point of the path. Maximization of $H$ leads to two formulas. The first expresses optimal control $T^{\prime}$ in terms of $T$ and $z=-\partial V / \partial T$. For the linear kinetics of Eq. (32) we obtain

$$
\frac{\partial V}{\partial T}-\frac{\partial f_{0}\left(T, T^{\prime}\right)}{\partial T^{\prime}}=\frac{\partial V}{\partial T}+c\left(1-\frac{T^{e} T}{T^{\prime 2}}\right)=0 .
$$

Whereas the second formula is the original equation (32) without maximizing operation

$$
\frac{\partial V}{\partial \tau}+\frac{\partial V}{\partial T}\left(T^{\prime}-T\right)+c\left(1-\frac{T^{e}}{T^{\prime}}\right)\left(T^{\prime}-T\right)=0 .
$$

To obtain optimal control function $T(z, T)$ one should solve the second equality in equation (41) in terms of $T$ '. The result is optimal Carnot control $T^{\prime}$ in terms of $T$ and $z=-\partial V / \partial T$,

$$
T^{\prime}=\left(\frac{T^{e} T}{1+c^{-1} \partial V / \partial T}\right)^{1 / 2} .
$$

This expression is next substituted into (42); the result is the nonlinear Hamilton-Jacobi equation

$$
-\frac{\partial V}{\partial \tau}+c T\left(\sqrt{1+c^{-1} \partial V / \partial T}-\sqrt{T^{e} / T}\right)^{2}=0
$$

which contains the energylike (extremum) Hamiltonian of the extremum process

$$
H\left(T, \frac{\partial V}{\partial T}\right)=c T\left(\sqrt{1+c^{-1} \partial V / \partial T}-\sqrt{T^{e} / T}\right)^{2} .
$$

Expressing extremum Hamiltonian (45) in terms of state variable $T$ and Carnot control $T^{\prime}$ yields an energylike function satisfying the following relation

$$
E(T, u)=f_{0}-u \frac{\partial f_{0}}{\partial u}=c T^{e} \frac{\left(T^{\prime}-T\right)^{2}}{T^{\prime 2}}
$$

$E$ is the Legendre transform of the work lagrangian $l_{0}=-f_{0}$ with respect to the rate $u=\mathrm{d} T / \mathrm{d} \tau$. Assuming the Hamiltonian value, say $h$, one can exploit the constancy of $H$ to eliminate $\partial V / \partial T$. Next combining equation $H=h$ with optimal control (43), or with an equivalent result for heat flow control $u=T^{\prime}-T$

$$
u=\left(\frac{T^{e} T}{1+c^{-1} \partial V / \partial T}\right)^{1 / 2}-T
$$

yields optimal rate $u=\dot{T}$ in terms of temperature $T$ and the Hamiltonian constant $h$

$$
\dot{T}=\left\{ \pm \sqrt{h / c T^{e}}\left(1- \pm \sqrt{h / c T^{e}}\right)^{-1}\right\} T .
$$

A more general form of this result which applies to systems with internal dissipation (factor $\Phi$ ) and applies to the pseudoNewtonian model of radiation is

$$
\dot{T}=\left( \pm \sqrt{\frac{h_{\sigma}}{\Phi c_{v}(T)}}\left(1- \pm \sqrt{\frac{h_{\sigma}}{\Phi c_{v}(T)}}\right)^{-1}\right) T \equiv \xi\left(h_{\sigma}, \Phi, T\right) T
$$

where $\xi$, defined in the above equation, is an intensity index and $h_{\sigma}=h / T$. This result is valid the temperature dependent heat capacity $c_{\mathrm{v}}(T)=4 a_{0} T^{3}$. Positive $\xi$ refer to heating of the resource fluid in the heat-pump mode, and the negative - to cooling of this fluid in the engine mode. Therefore pseudo-Newtonian systems produce power relaxing with the optimal rate

$$
\dot{T}=\xi\left(h_{\sigma}, T, \Phi\right) T \text {. }
$$

Equations (49) and (50) describe the optimal trajectory in terms of state $T$ and a parameter $h_{\sigma}$. The optimal Carnot control is

$$
T^{\prime}=\left(1+\xi\left(h_{\sigma}, \Phi, T\right)\right) T
$$


Comparing with linear systems, pseudo-Newtonian relaxation curve is not exponential. Optimal temperature of radiation downgraded in engine mode (upgraded in heat-pump mode) is illustrated in [4], [5] and [7]. HJB theory of energy systems can also be based on minimum properties of entropy production.

\section{Standardized Functions of Optimal Work}

When standard the standard exergy boundary conditions are applied optimal work functions become generalized (rate dependent exergies. Let us illustrate these for linear systems. Substituting temperature control (51) with a constant $\xi$ into work functional (30) and integrating along an optimal path subject the standard exergy boundary conditions yields an extremal work function

$$
V\left(T^{i}, T^{f}, h\right)=c\left(T^{i}-T^{f}\right)-c T^{e} \ln \frac{T^{i}}{T^{f}}-c T^{e} \sqrt{\frac{h}{c T^{e}}} \ln \frac{T^{i}}{T^{f}}
$$

This expression is valid for every process mode. Integration of Eq (49) subject to end conditions $T(\tau)=T^{\mathrm{i}}$ and $T\left(\tau^{\mathrm{f}}\right)=T^{\mathrm{f}}$ leads to $V$ in terms of the process duration.

For radiation $c_{\mathrm{v}}(T)=4 a_{0} T^{3}$, where $a_{0}$ is the radiation constant. An optimal path consistent with Eqs. (50) - (52) is of the form

$$
\pm(4 / 3) a_{0}{ }^{1 / 2} \Phi^{1 / 2} h_{\sigma}^{-1 / 2}\left(T^{3 / 2}-T^{i^{3 / 2}}\right)-\ln \left(T / T^{i}\right)=\tau-\tau^{i} .
$$

The integration limits refer to the initial state $(i)$ and a current state of the radiation fluid, i.e. temperatures $T$ and $T$ corresponding with $\tau$ and $\tau$. Optimal curve (53) refers to the radiation relaxation subject to constraint resulting from Eq. (50).

The corresponding extremal work function per unit volume of flowing radiation is

$$
\begin{aligned}
& V \equiv h_{v}^{i}-h_{v}^{f}-T^{e}\left(s_{v}^{i}-s_{v}^{f}\right)-(4 / 3) a_{0}{ }^{1 / 2} h_{\sigma}^{l / 2} \Phi^{1 / 2} T^{e}\left(T^{i^{3 / 2}}-T^{f^{3 / 2}}\right) \\
& +(4 / 3) a_{0} T^{e}(1-\Phi)\left(T^{i^{3}}-T^{f^{3}}\right)
\end{aligned}
$$

The generalized exergy of radiation at flow [14] follows from Eq. (54) after applying exergy boundary conditions. Yet the classical exergy of radiation at flow resides in the exergy equation in Jeter's form [15] rather than in Petela's form [14].

\section{Chemical Power Systems}

The developed approach can be extended to chemical and electrochemical engines. Here we shall make only a few basic remarks. In chemical engines mass transports participate in transformation of chemical affinities into mechanical power [12], [16]. Yet, as opposed to thermal machines, in chemical ones generalized streams or reservoirs are present, capable of providing both heat and substance. Large streams or infinite reservoirs assure constancy of chemical potentials. Problems of extremum power (maximum of power produced and minimum of power consumed) are static optimization problems. For a finite "upper stream", however, amount and chemical potential of an active reactant decrease in time, and considered problems are those of dynamic optimization and variational calculus. Because of the diversity and complexity of chemical systems the area of power producing chemistries is extremely broad. The simplest model of power producing chemical engine is that with an isothermal isomerization reaction, $A_{1}+A_{2}=0$ [3],[12]. Power expression and efficiency formula of a chemical system follow from the entropy conservation and energy balance of a powerproducing zone ('active part'). In an 'endoreversible chemical engine' total entropy flux is continuous through the active zone. When a formula describing this continuity is combined with energy balance we find in an isothermal case

$$
p=\left(\mu_{1^{\prime}}-\mu_{2^{\prime}}\right) n_{1},
$$

where the feed flux $n_{1}$ equals to $n$, an invariant molar flux of reagents. Process efficiency $\zeta$ is defined as power yield per flux $n$. This efficiency is identical with the chemical affinity of our reaction in the chemically active part of the system. While $\zeta$ is not dimensionless, it describes correctly the system. In terms of Carnot variable, $\mu$, which satisfies Eq. (27)

$$
\zeta=\mu^{\prime}-\mu_{2} \text {. }
$$

For a steady engine the following function describes chemical Carnot control $\mu$ ' in terms of fuel flux $n_{1}$ and its mole fraction $x$

$$
\mu^{\prime}=\mu_{2}+\zeta_{0}+R T \ln \left(\frac{x_{1}-n_{1} g_{1}^{-1}}{n_{1} g_{2}^{-1}+x_{2}}\right)
$$

As Eq. (56) is valid, Eq. (57) also characterizes the efficiency control in terms of $n$ and fuel fraction $x$.

Equation (57) shows that an effective concentration of the reactant in upper reservoir $x_{\text {leff }}=x_{1}-g_{1}^{-1} n$ is decreased, whereas an effective concentration of the product in lower reservoir $x_{2 \text { eff }}=x_{2}+g_{2}^{-1} n$ is increased due to the finite mass flux. Therefore efficiency $\zeta$ decreases nonlinearly with $n$. When effect of resistances $\left(g_{\mathrm{k}}\right)^{-1}$ is ignorable or flux $n$ is very small, reversible Carnot-like chemical efficiency, $\zeta_{C}$, is attained. The power function, described by the product $\zeta(n) n$, exhibits a maximum for a finite value of the fuel flux, $n$.

Application of Eq. (57) to an unsteady case yields a functional

$$
W=-\int_{\tau_{1}^{i}}^{\tau_{1}^{f}}\left\{\zeta_{0}+R T \ln \left(\frac{X /(1+X)+d X / d \tau_{1}}{x_{2}-j d X / d \tau_{1}}\right)\right\} \frac{d X}{d \tau_{1}} d \tau_{1}
$$

whose maximum describes the dynamical limit of the system. Here $\mathrm{X}=\mathrm{x} /(1-\mathrm{x})$ and $j$ equals the ratio of upper to lower mass conductance, $g_{1} / g_{2}$. The path optimality condition may be expressed in terms of the constancy of the Hamiltonian

$$
H(X, \dot{X})=R T \dot{X}^{2}\left(\frac{1+X}{X}+\frac{j}{x_{2}}\right) \text {. }
$$

For low rates and large concentrations $X$ (mole fractions $\mathrm{x}_{1}$ close to the unity) optimal relaxation rate of the fuel resource is approximately constant. Yet, in an arbitrary situation optimal rates are state dependent so as to preserve the constancy of $H$ in Eq. (59). Extensions of Eq. (57) are known for multireaction systems and those with internal imperfections [17].

\section{Fuel Cells at Steady States}

Now we consider performance bounds in fuel cells. These systems are electrochemical flow engines propelled by chemical reactions, which satisfy requirements imposed by chemical stoichiometry. Units which produce power are engines whereas those which consume power are electrolyzers. Their main 
advantage in comparison to heat engines is that their efficiency is not a major function of device size. Figure 3 illustrates a solid oxide fuel cell (SOFC) which works in the power yield mode.

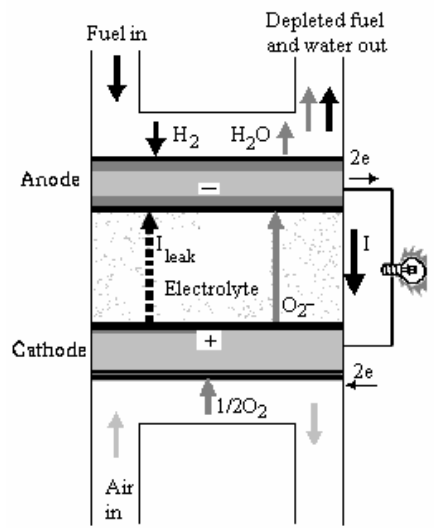

Fig. 3. Principle of a solid oxide fuel cell

The basic structure of fuel cells includes electrolyte layer in contact with a porous anode and cathode on either side. Gaseous fuels are fed continuously to the anode (negative electrode) compartment and an oxidant (i.e., oxygen from air) is fed to the cathode (positive electrode) compartment. Electrochemical reactions at the electrodes produce an electric current. The effect is the oxidation of fuel, e.g. hydrogen, and reduction of oxidant, e.g. oxygen. This makes fuel cells similar to an engine in Fig. 1.

Voltage lowering in fuel cells below the reversible value is a good measure of their imperfection, Fig.4. With the concept of effective nonlinear resistances operating voltage of a fuel cell can be represented as the departure from the ideal voltage $E^{0}$

$$
V=E^{O}-V_{\text {int }}=E-V_{\text {act }}-V_{\text {conc }}-V_{\text {ohm }}
$$

The losses, which are called polarization, include three main sources: activation polarization ( $\left.V_{\text {act }}\right)$, ohmic polarization $\left(V_{\text {ohm }}\right)$, and concentration polarization $\left(V_{\text {conc }}\right)$. Large number of approaches for calculating polarization losses has been reviewed [18]. Activation and concentration polarization occurs at both anode and cathode locations, while the resistive polarization represents ohmic losses throughout the fuel cell. As the voltage losses increase with current, the initially increasing power finally decreases for sufficiently large currents, so that maxima of power are observed (Fig. 4).

The voltage equation used in Wierzbicki's thesis [18] for the purpose of the calculation of fuel cell power is:

$$
V=E_{0}\left(T, p_{H_{2}}\right)-i A_{R}\left(p_{H_{2}}\right) \exp \left(\frac{\Delta E}{R T}\right)+B \ln \left(1-\frac{i}{i_{L}\left(T, p_{H_{2}}\right)}\right)
$$

where a limiting current is introduced defined by an equation

$$
i_{L}=C_{1} T^{-1} \exp \left(\frac{-E_{a}}{R T}\right) p_{H_{2}}
$$

in which $C_{1}$ is a experimentally determined parameter. Power density is simply the product of voltage $V$ and current density $i$. In an ideal situation (no losses) the cell voltage is defined by the Nernst equation. Yet, while the first term of Eq. (61) defines the voltage without load, it nonetheless takes into account losses of the so-called idle run, which are the effect of flaws in electrode constructions and other imperfections which cause that the open circuit voltage will in reality be lower than the theoretical value. Activation polarization $V_{\text {act }}$ is neglected in this model. The losses include ohmic and concentration polarization. The second term of Eq. (61) quantifies ohmic losses associated with electric resistance of electrodes and flow resistance of ions through the electrolyte. The third term refers to mass transport losses. Quantity $i_{L}$ is the particular current arising when the fuel is consumed in the reaction with the maximum possible feed rate.

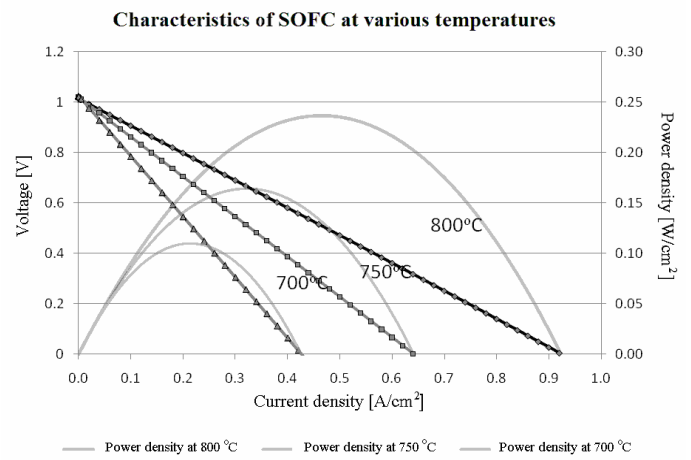

Fig.4. Voltage-current density and power-current density characteristics of the SOFC for various temperatures. Continuous lines represent the Aspen Plus ${ }^{\mathrm{TM}}$ calculations testing the model consistency with the experiments. These lines were obtained in Wierzbicki's MsD thesis supervised by S. Sieniutycz and J. Jewulski [19]. Points refer to experiments of Wierzbicki and Jewulski in Warsaw Institute of Energetics (Wierzbicki, [19], and his ref 18).

In the literature there are many experimental and theoretical examples showing power maxima in fuel cells and proving the suitability of the theory to chemical and electrochemical systems. For example, data obtained in L. Chen's research group [18] are consistent with those of Wierzbicki [19].

\section{Concluding Remarks}

This research provides data for power production bounds (limits) which are enhanced in comparison with those predicted by the classical thermodynamics. In fact, thermostatic bounds are often too far from reality to be really useful. Generalized bounds, obtained here by solving HJB equations, are stronger than those predicted by thermostatics. As opposed to classical thermodynamics, they depend not only on state changes but also on process irreversibilities, ratios of stream flows, stream directions, and mechanism of heat and mass transfer. The methodology familiar for thermal machines has been extended here to chemical and electrochemical engines. Extensions are also available for multicomponent, multireaction units [17]).

A real work supply can only be larger than the finite-rate bound obtained by the optimization. Similarly, the real work delivered from a nonequilibrium work-producing system (with the same boundary states and duration but with a suboptimal control) can only be lower than the corresponding finite-rate bound. Indeed, the two bounds, for a process and its inverse, which coincide in thermostatics, diverge in thermodynamics at a rate that grows 
with any index quantifying process deviation from the static behavior, e.g. Hamiltonian $H$. With thermokinetic models, we can confront and surmount the limitations of applying classical thermodynamic bounds to real processes. This is a direction with many opportunities, especially for separation and chemical systems. Electrochemical systems are especially important in view of their clean, efficient, and reliable performance.

\section{Acknowledgments}

This research was supported in part by Polish Ministry of Science, grant NN208 019434: Thermodynamics and Optimization of Chemical and Electrochemical Energy Generators with Applications to Fuel Cells. Results in Fig. 4 were found with M. Wierzbicki during the present author's supervising of his MsD thesis. Thesis co-supervising by $\mathrm{dr}$ J. Jewulski of Warsaw Institute of Energetics is also appreciated.

\section{Nomenclature}

$a_{\mathrm{v}}$ total area of energy exchange per unit volume $\left[\mathrm{m}^{-1}\right]$

$E^{0}, E_{0}$ Nernst ideal voltage and idle run voltage, respectively [V]

$\dot{G}$ resource flux $\left[\mathrm{gs}^{-1}, \mathrm{mols}^{-1}\right]$

$g_{1}, g$ partial and overall conductance $\left[\mathrm{Js}^{-1} \mathrm{~K}^{-\mathrm{a}}\right]$

$H$ Hamiltonian function

$H_{\mathrm{TU}}$ height of transfer unit [m]

$h$ numerical value of Hamiltonian $\left[\mathrm{Jm}^{-3} \mathrm{~K}^{-1}\right]$

$h, h_{\mathrm{v}}$ specific and volumetric enthalpies $\left[\mathrm{Jg}^{-1}, \mathrm{Jm}^{-3}\right]$

$i$-electric current density $\left[\mathrm{Am}^{-2}\right]$

$n$ flux of fuel reagents $\left[\mathrm{gs}^{-1}, \mathrm{mols}^{-1}\right]$

$p=\dot{W}$ power output $\left[\mathrm{Js}^{-1}\right]$

$p_{m}^{0}$ molar constant of photons density $\left[\mathrm{molm}^{-2} \mathrm{~K}^{-3} \mathrm{~s}^{-1}\right]$

$q$ heat flux between a stream and power generator $\left[\mathrm{Js}^{-1}\right]$

$Q$ total heat flux involving transferred entropies $\left[\mathrm{Js}^{-1}\right]$

$S, S_{\sigma}$ entropy and entropy produced $\left[\mathrm{JK}^{-1}\right]$

$T$ variable temperature of resource $[\mathrm{K}]$

$T_{1}, T_{2}$ bulk temperatures of reservoirs 1 and $2[\mathrm{~K}]$

$T^{\prime}$ Carnot temperature control $[\mathrm{K}]$,

$t$ physical time [s]

$u$ and $v$ rate controls, $d T / d \tau$ and $\mathrm{d} T / \mathrm{d} t,\left[\mathrm{~K}, \mathrm{Ks}^{-1}\right]$

$V$ voltage, maximum work function, resp. [V, $\left.\mathrm{Jmol}^{-1}\right]$

$W$ work produced, positive in engine mode [J]

$x$ mass fraction [-], length coordinate [m]

\section{Greek symbols}

$\alpha_{1}, \alpha^{\prime}$ partial and overall heat coefficients $\left[\mathrm{Jm}^{-2} \mathrm{~s}^{-1} \mathrm{~K}^{-1}\right]$

$\beta$ effective coefficient of radiation transfer related to constant $p_{m}^{0}$ and

Stefan-Boltzmann constant $\left(\beta=\sigma a_{v} c_{h}^{-1}\left(p_{m}^{0}\right)^{-1}\right)\left[\mathrm{s}^{-1}\right]$

$\varepsilon$ total energy flux, conservative along a conductor $\left[\mathrm{Js}^{-1}\right]$

$\eta=p / q_{1}$ first-law thermal efficiency [-]

$\chi=\rho c_{v}\left(\alpha a_{\mathrm{v}}\right)^{-1}$ time constant [s]

$\mu$ chemical potential $\left[\mathrm{Jmol}^{-1}\right]$

$\mu^{\prime}$ Carnot chemical potential $\left[\mathrm{Jmol}^{-1}\right]$

$\Phi$ factor of internal irreversibility [-]

$\sigma$ Stefan-Boltzmann constant for radiation $\left[\mathrm{Jm}^{-2} \mathrm{~s}^{-1} \mathrm{~K}^{-4}\right]$

$\sigma_{\mathrm{s}}$ entropy production rate in the system $\left[\mathrm{JK}^{-1} \mathrm{~s}^{-1}\right]$

$\xi$ intensity index [-]

$\zeta$ chemical efficiency [-]

$\tau$ dimensionless time or number of transfer units [-]

\section{Subscripts}

$C$-Carnot point; $m$ - molar; $-v$ per unit volume; 0 idle run voltage

\section{Superscripts}

$e$ environment; $i$ initial state; $f$ initial state; 0 ideal voltage

\section{References}

[1] Sieniutycz, S., 2007. "Limis for production or consumption of energy and substances predicted by thermodynamics", International conference on renewable energy and power quality (ICREPQ'07), Sevilla, Spain, 28-30 March, http://www.icrepq.com, paper 380.

[2] Curzon, F.L., Ahlborn, B., 1975. "Efficiency of Carnot engine at maximum power output". American J. Phys., 43(1), 22-24.

[3] De Vos, A., 1994. Endoreversible Thermodynamics of Solar Energy Conversion, Oxford University Press, pp. 30-41.

[4] Sieniutycz, S., Kuran, P., 2005. "Nonlinear models for mechanical energy production in imperfect generators driven by thermal or solar energy", Intern. J. Heat Mass Transfer, 48(3-4), 719-730.

[5] Sieniutycz, S., Kuran, P., 2006. "Modeling thermal behavior and work flux in finite-rate systems with radiation". Intern. J. Heat and Mass Transfer, 49(17-18), 3264-3283.

[6] Sieniutycz, S., 2009. "Dynamic programming and Lagrange multipliers for active relaxation of resources in non-equilibrium systems. Applied Mathematical Modeling, 33(3), 1457-1478.

[7] Kuran, P., 2006. Nonlinear Models of Production of Mechanical Energy in Non-Ideal Generators Driven by Thermal or Solar Energy, Thesis (PhD). Warsaw University of Technology.

[8] Berry, R. S., Kazakov, V. A., Sieniutycz, S., Szwast, Z., Tsirlin, A. M., 2000. Thermodynamic Optimization of Finite Time Processes, Chichester, Wiley, p.197.

[9] Sieniutycz, S., 2003. "A synthesis of thermodynamic models unifying traditional and work-driven operations with heat and mass exchange". Open Sys. \& Information Dynamics, 10(1), 3149.

[10] Sieniutycz, S., 2003. "Carnot controls to unify traditional and work-assisted operations with heat \& mass transfer". International Journal of Applied Thermodynamics, 6(2), 59-67.

[11] Sieniutycz, S., Jeżowski, J., 2009. Energy Optimization in Process Systems. Oxford: Elsevier.

[12] Sieniutycz, S., 2008. "An analysis of power and entropy generation in a chemical engine". Intern. J. of Heat and Mass Transfer, 51(25-26), 5859-5871.

[13] Bellman, R., 1961. Adaptive Control Processes: a Guided Tour. Princeton University Press.

[14] Petela, R., 1964. "Exergy of heat radiation". J. Heat Transfer, 86(2), 187-192.

[15] Jeter, J., 1981. "Maximum conversion efficiency for the utilization of direct solar radiation". Solar Energy, 26(3), 231-236.

[16] Tsirlin, A. M., Kazakov, V., Mironova, V. A., Amelkin, S. A., 1998. "Finite-time thermodynamics: conditions of minimal dissipation for thermodynamic process". Physical Review E, 58 (1), 215-223.

[17] Sieniutycz, S., 2009. "Complex chemical systems with power production driven by mass transfer". Intern. J. of Heat and Mass Transfer, 52(10), 2453-2465.

[18] Zhao, Y., Ou, C., Chen, J., 2008. "A new analytical approach to model and evaluate the performance of a class of irreversible fuel cells". International Journal of Hydrogen Energy, 2008 (33), 4161- 4170.

[19] Wierzbicki, M., 2009. Optimization of SOFC based energy system using Aspen Plus ${ }^{T M}$, Thesis (MsD) supervised by S. Sieniutycz (Faculty of Chemical and Process Engineering, Warsaw University of Technology) and J. Jewulski (Laboratory of Fuel Cells, Institute of Energetics, Warsaw). 